\title{
A novel translational control mechanism involving RNA structures within coding sequences
}

\author{
Jennifer Jungfleisch, ${ }^{1}$ Danny D. Nedialkova, ${ }^{2,3,10}$ Ivan Dotu, ${ }^{4,10}$ Katherine E. Sloan, ${ }^{5}$ \\ Neus Martinez-Bosch, ${ }^{6}$ Lukas Brüning, ${ }^{5}$ Emanuele Raineri, ${ }^{7}$ Pilar Navarro, ${ }^{6}$ \\ Markus T. Bohnsack, ${ }^{5,8}$ Sebastian A. Leidel, ${ }^{2,3,9}$ and Juana Díez ${ }^{1}$ \\ ${ }^{1}$ Molecular Virology Group, Department of Experimental and Health Sciences, Universitat Pompeu Fabra, 08003 Barcelona, Spain; \\ ${ }^{2}$ Max Planck Research Group for RNA Biology, Max Planck Institute for Molecular Biomedicine, 48149 Münster, Germany; \\ ${ }^{3}$ Cells-in-Motion Cluster of Excellence, University of Münster, 48149 Münster, Germany; ${ }^{4}$ Research Programme on Biomedical \\ Informatics (GRIB), Department of Experimental and Health Sciences, Universitat Pompeu Fabra, Hospital del Mar Medical Research \\ Institute (IMIM), 08003 Barcelona, Spain; ${ }^{5}$ Institute for Molecular Biology, Göttingen University Medical Department, 37073 \\ Göttingen, Germany; ${ }^{6}$ Program of Cancer Research, Hospital del Mar Medical Research Institute (IMIM), 08003 Barcelona, Spain; \\ ${ }^{7}$ Statistical Genomics, Centro Nacional de Analisis Genomica, 08028 Barcelona, Spain; ${ }^{8}$ Göttingen Center for Molecular Biosciences, \\ Georg-August University, 37073 Göttingen, Germany; ${ }^{9}$ Faculty of Medicine, University of Münster, 48149 Münster, Germany
}

\begin{abstract}
The impact of RNA structures in coding sequences (CDS) within mRNAs is poorly understood. Here, we identify a novel and highly conserved mechanism of translational control involving RNA structures within coding sequences and the DEADbox helicase Dhhl. Using yeast genetics and genome-wide ribosome profiling analyses, we show that this mechanism, initially derived from studies of the Brome Mosaic virus RNA genome, extends to yeast and human mRNAs highly enriched in membrane and secreted proteins. All Dhhl-dependent mRNAs, viral and cellular, share key common features. First, they contain long and highly structured CDSs, including a region located around nucleotide 70 after the translation initiation site; second, they are directly bound by Dhhl with a specific binding distribution; and third, complementary experimental approaches suggest that they are activated by Dhhl at the translation initiation step. Our results show that ribosome translocation is not the only unwinding force of CDS and uncover a novel layer of translational control that involves RNA helicases and RNA folding within CDS providing novel opportunities for regulation of membrane and secretome proteins.
\end{abstract}

[Supplemental material is available for this article.]

Structural features in messenger RNA (mRNA) regulate its localization, translation, and degradation. The dynamic folding of mRNA into secondary and tertiary structures guides the interaction with proteins and RNAs that ultimately directs mRNA fates. Yet, the elucidation of such structures and their functional implications remain elusive. Recent advances in next-generation sequencing (NGS) in combination with nuclease treatments or chemical probing allowed, for the first time, experimental genome-wide measurements of RNA secondary structures. These seminal studies have uncovered novel evolutionary conserved structural patterns and their nexus to translational control (Mortimer et al. 2014). A surprising discovery was that the CDSs of Saccharomyces cerevisiae mRNAs present a higher degree of secondary structures than untranslated regions (UTRs) in vitro (Kertesz et al. 2010). Subsequent studies demonstrated that CDSs are significantly less structured or, alternatively, more structurally dynamic in vivo than in vitro (Rouskin et al. 2014). This remodeling is energy-dependent, as depletion of ATP results in recovery of the structure, mimicking the in vitro results. How this in vivo remodeling is achieved and what functional implications it represents are poorly understood. Ribosome translocation during translation elongation has been

\footnotetext{
${ }^{10}$ These authors contributed equally to this work. Corresponding author: juana.diez@upf.edu Article published online before print. Article, supplemental material, and publication date are at http://www.genome.org/cgi/doi/10.1101/gr.209015.116.
}

traditionally considered to drive CDS unwinding since strand separation activity is inherent to the ribosome, requiring no exogenous helicases (Takyar et al. 2005). However, this activity might not be the only unwinding force in vivo, as high ribosome occupancy within coding regions is not associated with lower structure propensities (Rouskin et al. 2014).

Strong candidates for factors directing mRNA unfolding in vivo are ATP-dependent helicases. From these, the large DEADbox family of RNA helicases is involved in all cellular processes that require RNA remodeling, such as transcription, pre-mRNA splicing, ribosome biogenesis, and RNA decay (Martin et al. 2013; Jarmoskaite and Russell 2014). In addition to ATP-dependent RNA unwinding activities, DEAD-box RNA helicases promote RNA duplex formation, serve as assembly platforms for the formation of large RNP complexes, and displace proteins from RNA (Linder and Jankowsky 2011). Dhh1 is a highly evolutionarily conserved member of the family of DEAD-box RNA helicases. The conservation is such that, in yeast, Dhh1 can be functionally replaced by its counterparts from Drosophila melanogaster (Me31B), Xenopus laevis (Xp54), or humans (DDX6) (Maekawa et al. 1994; Tseng-

(c) 2017 Jungfleisch et al. This article is distributed exclusively by Cold Spring Harbor Laboratory Press for the first six months after the full-issue publication date (see http://genome.cshlp.org/site/misc/terms.xhtml). After six months, it is available under a Creative Commons License (Attribution-NonCommercial 4.0 International), as described at http://creativecommons.org/licenses/ by-nc/4.0/. 
Rogenski et al. 2003; Westmoreland et al. 2003; Alves-Rodrigues et al. 2007). Dhh1 functions as a translational repressor and as a decapping activator in the major deadenylation-dependent $5^{\prime}-3^{\prime}$ mRNA decay pathway (Presnyak and Coller 2013). Former in vitro studies suggested that Dhh1 represses mRNA translation by acting on translation initiation and translation elongation (Coller and Parker 2005; Franks and Lykke-Andersen 2008; Sweet et al. 2012). In humans, DDX6 functions as well in promoting miRNA-mediated repression via association with AGO1, AGO2, and the CCR4-NOT complex (Chu and Rana 2006; Chen et al. 2014; Mathys et al. 2014).

In contrast to the well-established Dhh1 repressing functions, we and others have found that Dhh1 activates translation of viral RNA genomes. By using a model system that allows the replication of the plant Brome mosaic virus (BMV) in yeast, we have previously shown that Dhh1 depletion dramatically inhibits BMV RNA translation (Alves-Rodrigues et al. 2007). Moreover, this role is extended to its human homolog DDX6 since first, in the yeast model system it replaces Dhh1 to promote BMV RNA translation, and second, it promotes translation of the human Hepatitis C virus (HCV) RNA genome in hepatoma cell lines (Alves-Rodrigues et al. 2007; Scheller et al. 2009; Huys et al. 2013). The mechanism for translational activation by Dhh1 is unclear, but it should not involve the cap structure because, unlike BMV RNA, HCV RNA is translated via an IRES-dependent mechanism. BMV and HCV belong to the large group of positive-strand RNA viruses whose RNA genomes are highly structured. Throughout infection, positive-strand RNA genomes display three mutually exclusive functions. They first function as mRNAs for expression of the viral proteins, later as templates for RNA replication, and subsequently as genomes for encapsidation of new particles. Profound remodeling steps of the viral ribonucleoprotein complex are temporarily required to coordinate these essential transitions that are poorly understood. Given the dynamic folding properties of viral RNA genomes and the fact that viruses hijack processes already existing in the host, we hypothesized that the positive role of Dhh1 in viral RNA translation is linked to specific RNA folding regulations that may be extended to a specific set of cellular mRNAs. By combining viral studies in the BMV/yeast system with high-throughput RNA-seq, ribosome profiling and CRAC (UV crosslinking and analysis of cDNA) of host mRNAs, here, we uncover an additional layer of translational control involving an RNA helicase and RNA folding within CDSs that is seemingly conserved from yeast to humans and hijacked by viruses.

\section{Results}

\section{Dhhl ATPase activity promotes translation of BMV RNA2}

Dhh1 has been well described as a translational repressor and a decapping activator; however, by using the ability of BMV RNA to translate and replicate in the yeast Saccharomyces cerevisiae, we have previously shown that Dhh1 promotes translation of the BMV RNA2 (Mas et al. 2006). BMV RNA2 is $5^{\prime}$-capped and contains a tRNA-like structure instead of a poly(A)-tail at the $3^{\prime}$ end (Noueiry and Ahlquist 2003). To elucidate which Dhh1 features are required for this unexpected function, we introduced several characterized point mutations in multiple conserved motifs (Cheng et al. 2005; Dutta et al. 2011) and studied their in vivo effect on both yeast viability and BMV RNA2 translation (Fig. 1). The mutants were named based on the mutated motif (described in Fig. 1A; Supplemental Fig. S1A). A single-copy plasmid expressing each mutant

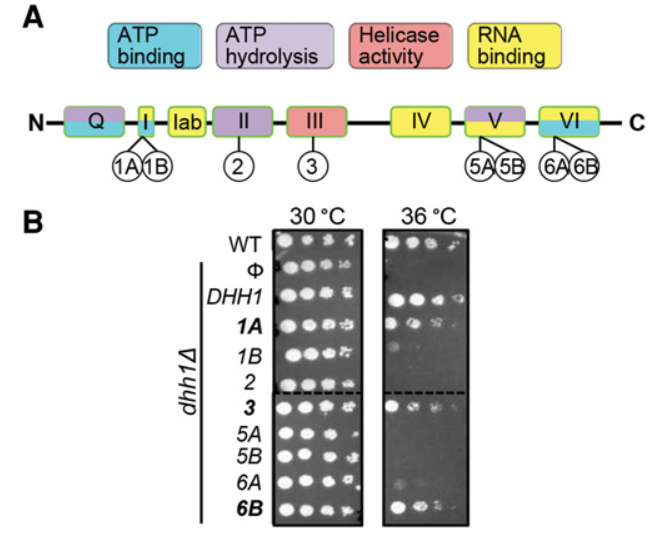

C

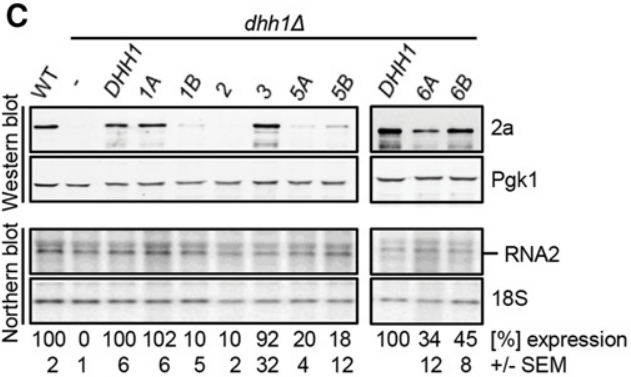

D

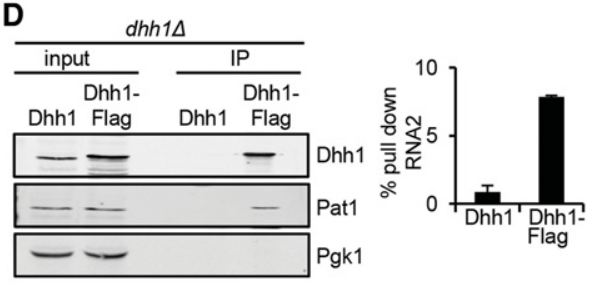

$\mathbf{E}$

\begin{tabular}{|c|c|c|c|c|c|c|c|c|}
\hline \multicolumn{4}{|c|}{ WT } & \multicolumn{4}{|c|}{$d h h 1 \Delta$} & \\
\hline 0 & 10 & 20 & 60 & 0 & 10 & 20 & 60 & $\min$ \\
\hline$\Longrightarrow$ & $=$ & 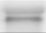 & - & $\equiv$ & $=$ & $\Longrightarrow$ & 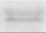 & - RNA2 \\
\hline- & - & - & - & - & - & - & - & $18 \mathrm{~S}$ \\
\hline
\end{tabular}

Figure 1. Effect of multiple Dhh1 mutations on cell growth and on viral RNA translation. (A) Motifs and functional domains of the DEAD-box RNA helicase Dhh1. Numbers $1 \mathrm{~A}$ to $6 \mathrm{~B}$ indicate the location of the point mutations. (B) Growth at $30^{\circ} \mathrm{C}$ and $36^{\circ} \mathrm{C}$ of WT and $d h h 1 \Delta$ expressing various Dhh1 mutant alleles. (C) Effect of Dhh1 mutants on BMV RNA2 translation. The $d h h 1 \Delta$ strain was transformed with a plasmid expressing RNA2 together with an empty plasmid or a plasmid expressing $\mathrm{DHH} 1$ or the different dhh1 mutant alleles. RNA2 and protein 2a were analyzed by Northern and Western blot, respectively. As a control for equal loading and sample quality, 18S rRNA and phosphoglycerate kinase protein 1 (Pgk1) were also analyzed. The average $2 \mathrm{a}$ expression value from at least three independent experiments is shown. The average value obtained for cells expressing $D H H 1$ was set to 100 . The SEM values are indicated below. (D) Dhh1 binds BMV RNA2. Western blot analysis of immunoprecipitation assays carried out in dhh1 $1 \Delta$ cells expressing RNA2 and Dhh1 or Dhh1-Flag. Input corresponds to $100 \mu \mathrm{g}$ of total protein present in lysates prior to precipitation and IP corresponds to the corresponding eluates from the anti-Flag matrix following precipitation. Diagram shows the relative amount ( \pm SEM) of precipitated RNA2 detected by qPCR after immunoprecipitation; the input amount of RNA2 was set to 100. (E) The halflife of RNA2 is not significantly altered in $d h h 1 \Delta$ cells. RNA2 was expressed from a GAL1 promoter in WT and $d h h 1 \Delta$ cells. Transcription was stopped by adding glucose at time point $t=0 \mathrm{~min}$. Samples were taken at different time points and RNA2 accumulation analyzed by Northern blot. Numbers below indicate the average half-life of RNA2 ( \pm SEM) based on three independent experiments. $T$-test analysis shows that the half-life is not significantly altered in $d h h 1 \Delta$ cells.

\section{Genome Research}

www.genome.org 
from the endogenous $D H H 1$ promoter was transformed into the $d h h 1 \Delta$ yeast strain. Expression levels of all Dhh1 mutant proteins were similar to that of wild-type Dhh1 (Supplemental Fig. S1B). First, we examined the effect of Dhh1 mutations on cell viability by studying cell growth at $30^{\circ} \mathrm{C}$ and $36^{\circ} \mathrm{C}$ (Fig. 1B). Confirming previous results for other yeast genetic backgrounds (Cheng et al. 2005; Dutta et al. 2011), all mutants failed to complement the dhh1 $1 \Delta$ thermo-sensitive phenotype, except mutant $1 \mathrm{~A}, 3$, and $6 \mathrm{~B}$, which are described not to affect Dhh1 remodeling activity (Cheng et al. 2005; Dutta et al. 2011). Importantly, a similar pattern was observed when we tested the effect of the Dhh1 mutants on BMV RNA2 translation (Fig. 1C). As steady-state BMV RNA2 levels were not affected, we conclude that the observed differences in 2a expression are due to translation defects. Together, these results indicate that similar Dhh1 features, including the ATPase activity, are required for both cell viability and BMV RNA2 translation.

A plausible explanation for these effects is that Dhh1 promotes translation of BMV RNA2 by remodeling its structure. If so, Dhh1 and RNA2 must interact. To test this possibility, we carried out RNA-co-immunoprecipitation experiments (RIP) using a Flagtagged Dhh1 (Fig. 1D). Addition of the Flag-tag did not affect Dhh1 function in BMV RNA2 translation (Supplemental Fig. S1C). As a control, Pat1, a known Dhh1-interacting protein (Coller et al. 2001; Nissan et al. 2010), was co-immunoprecipitated (Fig. 1D). BMV RNA2, detected by quantitative PCR, was enriched approximately ninefold in Dhh1-Flag eluates compared to the untagged Dhh1 control, indicating that Dhh1 interacts with viral RNA2 (Fig. 1D). To test whether the detected interaction is direct or mediated by another protein, we used CRAC analysis (Bohnsack et al. 2009, 2012). This method allows for a rapid and accurate identification of protein binding sites on RNA. Dhh1 directly bound at three sites in the CDS and one in the $3^{\prime}$ UTR, namely within the tRNA-like structure (Supplemental Fig. S1D). Given that Dhh1 is a decapping activator and BMV RNA2 is 5' -capped, the detected binding might be related to decapping and not to the translation activation mechanism. To rule this out, we determined whether Dhh1 affects the stability of RNA2, as expected should decapping be operative. BMV RNA2 was expressed in WT and $d h h 1 \Delta$ strains from an inducible $G A L$ promoter whose activity is repressed in the presence of glucose in the media. After adding glucose, RNA2 levels were followed over $60 \mathrm{~min}$ by Northern blot analysis (Fig. 1E). The half-life of RNA2 in dhh1 $\Delta$ cells was not significantly affected when compared to that in WT cells, indicating that Dhh1 is not involved in BMV RNA2 decapping or decay. Altogether, the data suggest a direct role of Dhh1 and its ATPase activity in BMV RNA2 translation.

\section{Depletion of Dhh1 shifts BMV RNA2 toward fractions containing single ribosomal subunits}

To determine which step of BMV RNA2 translation is promoted by Dhh1, we carried out polysome profiling analyses in WT and dhh1 1 cells expressing BMV RNA2. Absorbance measurements indicated that the average monosome to polysome $(\mathrm{M} / \mathrm{P})$ ratio in WT and $d h h 1 \Delta$ cells was similar, showing that the absence of Dhh1 does not affect polysome profiles, as previously reported (Fig. 2A; Coller and Parker 2005). Northern blot of RNA2 along the polysome profile showed that depletion of Dhh1 shifted RNA2 toward monosomal, 60S, and 40S fractions (Fig. 2B; Supplemental Fig. S2).

Puromycin, a drug that releases elongating ribosomes, is routinely used to confirm that mRNAs located in polysomal fractions are associated with ribosomes and not to merely heavy RNPs that do not contain ribosomes (Thermann and Hentze 2007). As
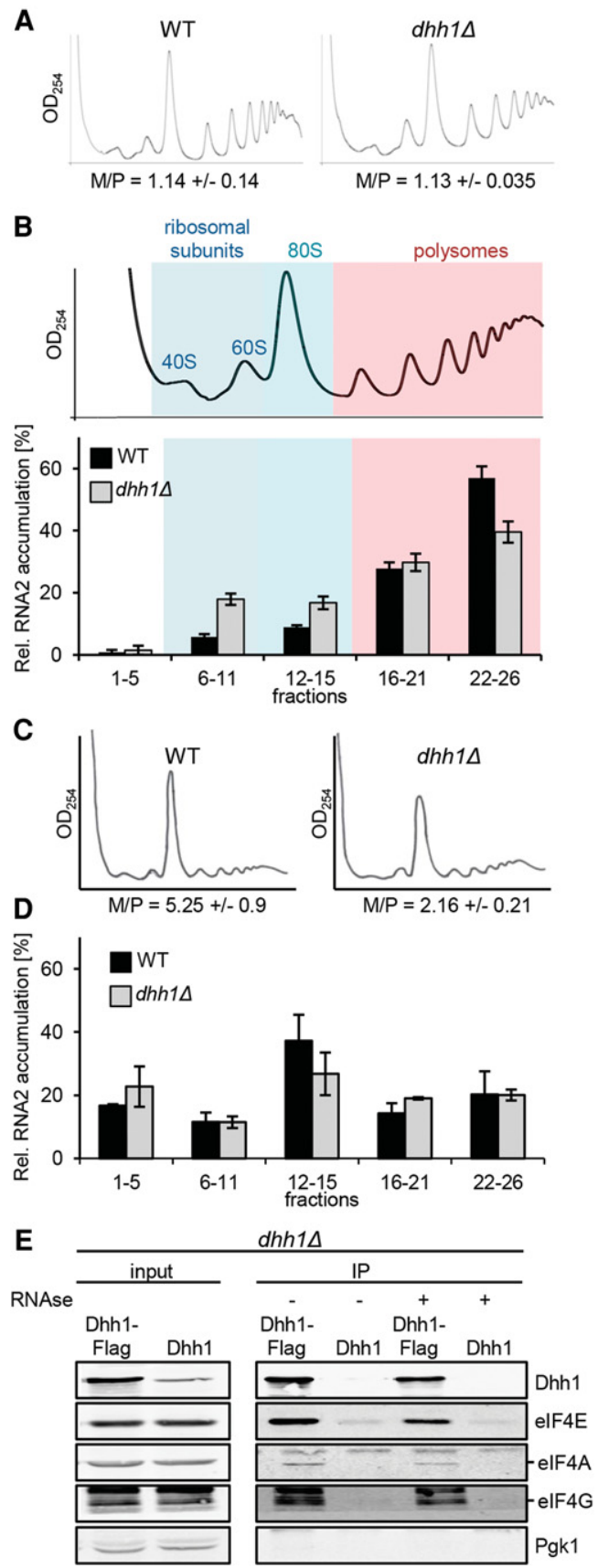

Figure 2. Depletion of Dhh1 shifts BMV RNA2 toward single ribosomal subunit fractions. $(A)$ Global translation is unaffected in a strain expressing RNA2. UV absorbance profile at $254 \mathrm{~nm}$ of an extract from WT and dhh1 cells expressing RNA2 after sedimentation on a 10\%-50\% sucrose gradient. The monosome to polysome ratio $(M / P \pm S E M)$ is not significantly affected. (B) Sucrose density gradient analysis of BMV RNA2 in WT and dhh1 $1 \Delta$ yeast. Below a representative UV absorbance profile is the distribution of normalized RNA2 levels in the specific fractions. Bars represent the average \pm SEM from three independent experiments. Fractions were grouped into free (1-5), single ribosomal subunits (6-11), monosomes (12-15), light polysomes (16-21), and heavy polysomes (22-26). The total amount of RNA2 recovered over the gradient was set to $100 \%$. (C) UV absorbance profile at $254 \mathrm{~nm}$ after 15-min glucose withdrawal of an extract from WT and $d h h 1 \Delta$ cells expressing RNA2. (D) Distribution of normalized RNA2 levels in the specific fractions after 15-min glucose withdrawal, as in $B$. (E) Dhh1 interacts with translation initiation factors. Western blot analysis of immunoprecipitation assays. Extracts were either treated (+) or not treated (-) with RNase A prior to the washing steps. 
puromycin and similar drugs do not work in yeast due to poor uptake (Melcher 1971; Schindler and Davies 1975), we inhibited global translation with 15 min of glucose withdrawal to test whether RNA2 is associated with ribosomes (Fig. 2C). Glucose deprivation inhibits translation initiation; thus, if RNA2 is associated with elongating ribosomes, a shift of RNA2 out of polysomes should be observed due to ribosome run-off and the inhibition of initiation. Glucose withdrawal led in WT and $d h h 1 \Delta$ cells to a comparable shift of RNA2 out of the polysomal fractions towards the free fractions and the monosomal fractions (Fig. 2D). Together, these results suggest that Dhh1 promotes translation initiation of RNA2. In agreement with this conclusion, immunoprecipitation analyses showed that Dhh1 binds to the translation initiation factors eIF4E, eIF4A, and eIF4G in an RNase-resistant manner (Fig. 2E).

\section{A stem-loop within the CDS of RNA2 confers dependence on Dhhl for translation}

To identify which BMV RNA2 regions confer Dhh1-dependence for translation, we replaced different RNA2 segments by alternative sequences and quantified $2 \mathrm{a}$ and RNA2 levels in the presence and absence of Dhh1 (Fig. 3A). The $5^{\prime}$ and 3' UTRs of BMV RNA2 are highly structured sequences with overlapping cis-acting signals essential for RNA2 translation and replication (Noueiry and Ahlquist 2003). The BMV 5' UTR was replaced by that of the GAL1 transcript and the nonpolyadenylated $3^{\prime}$ UTR by the polyadenylated 3' UTR of the ADH1 transcript. Translation of the natural RNA2 exhibited a 12 -fold dependence on Dhh1. Replacing either the $5^{\prime}$ UTR or the $3^{\prime}$ UTR decreased RNA2 Dhh1-dependence to twofold (Fig. 3A). Similarly, the concurrent replacement of both UTRs decreased Dhh1-dependence to twofold. Interestingly, replacing the CDS by that of GFP also resulted in a twofold Dhh1-dependence (Fig. 3A). An RNA derivative lacking RNA2 sequences did not depend on Dhh1 for translation, strengthening the specificity of Dhh1 for the viral RNA. Thus, full dependence on Dhh1 for RNA2 translation requires the concerted action of the 5' UTR, 3' UTR, and CDS.

To investigate how replacements of the different RNA2 segments affect Dhh1 binding, we carried out RIP in $d h h 1 \Delta$ cells expressing the different RNA2 constructs plus Dhh1-Flag or Dhh1. In line with the CRAC results indicating crosslinking of Dhh1 to the CDS and the 3' UTR of BMV RNA2, replacement of the CDS or the $3^{\prime}$ UTR region by the CDS of GFP or by the polyadenylated
3' UTR of the $A D H 1$ transcript reduced the amount of RNA2 co-immunoprecipitated, while replacement of the $5^{\prime}$ UTR region by that of the GAL1 transcript did not have any significant effect (Fig. 3B; Supplemental Fig. S3). Given that Dhh1 interacts with components of the cap-binding complex (Fig. 2E), it is likely that Dhh1 binds to both the $5^{\prime}$ UTR of BMV RNA2 and that of GAL1 mRNA through interactions with the cap-binding complex. However, such interaction would be only required for translation in the context of RNA2 sequences.

We next focused on the CDS because cis-acting sequences inside the CDS regulating translation have been rarely described. To investigate further which region of the CDS is responsible for

\section{Genome Research}

www.genome.org 
Dhh1-dependence, we generated a construct in which the CDS of RNA2 was fused to that of Renilla luciferase (RLUC) and obtained derivatives with successive deletions in the RNA2 CDS. The reporter construct containing the complete CDS of RNA2 (FLRLUC) exhibited a fivefold Dhh1-dependence for RLUC activity (Fig. 3C). Given the very low expression level of $2 \mathrm{a}$ protein in dhh1 $\Delta$ cells, the difference with the 12 -fold Dhh1-dependence observed for WT RNA2 is likely related to the higher sensitivity of luciferase measurements when compared to Western blot analysis. The 330-RLUC derivative shows a similar Dhh1-dependence to FL-RLUC, indicating that the cis-element conferring dependence on Dhh1 is located within these first $330 \mathrm{nt}$. Given that Dhh1 is a helicase, we explored whether there were structured elements within these $330 \mathrm{nt}$. Indeed, the Vienna RNA-fold package predicted the formation of a stem-loop structure at nt 42-85 after the initiation codon $(\Delta \mathrm{G}=-11.1 \mathrm{kcal} / \mathrm{mol})$ (Fig. 3D, left). To determine whether this stem-loop was responsible for Dhh1-dependence, we shortened the RNA2 CDS to 87 and 42 nt to generate constructs containing or lacking the stem-loop (Fig. 3C). While 87-RLUC maintained full Dhh1-dependence, this dependence was decreased to twofold in 42-RLUC (Fig. 3C). This twofold difference was also observed in a construct in which the complete RNA2 CDS was deleted (0-RLUC) and is mediated by the UTRs (Fig. 3C). The importance of nt 42 to 87 in translational regulation was also visualized when comparing RLUC activity of the different RNA2 derivatives in WT cells (Fig. 3E). Deletion of the 42-87 sequence led to a large increase of RLUC activity, indicating its repressing function. Nucleotides 87 to 330 also played an important role in translation; however, this effect was independent from Dhh1 activity (Fig. 3C). Collectively, these data indicate that nt 42 to 87 repress translation, a repression that is moderated by Dhh1. To determine whether the sequence or the predicted structure within nt 42-87 mediates Dhh1-dependence, we generated two RNA2 derivatives in which (1) the stem-loop was disrupted by replacing all bases on one side of the stem with complementary ones, and (2) the stem-loop was replaced by a structurally equivalent stem-loop with no sequence homology which was designed using RNAiFold (Fig. 3D, right; Garcia-Martin et al. 2013). Disruption of the stem-loop decreased Dhh1-dependence to that found in 0-RLUC (Fig. 3F). Importantly, Dhh1-dependence was recovered when the stem-loop was replaced by the designed one, as similar values were obtained with this and 87-RLUC RNA2, the derivative containing the minimal sequence conferring full Dhh1dependence. These data indicate that the stem-loop structure within the RNA2 CDS strongly inhibits translation and mediates the dependence on Dhh1 for translational stimulation.

\section{Dhhl drives translation of a selected set of cellular mRNAs}

Viruses are powerful tools to uncover cellular processes. In fact, most post-transcriptional processes were first described in viral studies and then confirmed in cellular mRNAs (Cullen 2009). To investigate whether Dhh1 can also drive translation of specific cellular mRNAs, we combined three high-throughput analyses in WT and dhh1s strains: RNA-seq, ribosome profiling, and CRAC (Fig. 4A). Ribosome profiling combined with RNA-seq provides a snapshot of the translational status of the genome on a transcriptome-wide level and is based on isolating and sequencing ribosome-protected fragments (Ingolia et al. 2012). We performed three independent ribosome profiling and RNA-seq experiments in $d h h 1 \Delta$ cells and were able to align sequence reads to 5424 genes of $S$. cerevisiae. Both ribosome profiling and RNA-seq results were highly reproducible between biological replicates for each strain $(r \approx 0.99)$. We compared these data to RNA-seq and ribosome profiling data from WT cells (GSE67387) (Nedialkova and Leidel 2015). Regarding the mRNA levels, in the absence of Dhh1 1467 genes did not exhibit significant changes, whereas 2177 have increased and 1780 decreased levels (Fig. 4B). Given the longestablished function of Dhh1 on mRNA decay, an increase in mRNA levels is expected, but a decrease might seem paradoxical. However, it is important to note that Dhh1 also plays a direct role in transcription of some mRNAs (Haimovich et al. 2013). The ribosome protected fragment (RPF) analysis shows that 1841 genes did not exhibit significant changes, while 1775 have decreased and 1808 increased ribosome occupancy (Fig. 4B). To study translation effects, we focused on transcripts where RPF levels, but not mRNA levels, are changed or where changes in the RPF and mRNA levels go in opposite directions. With this, we ensure that the selected mRNAs are translationally regulated. Following these criteria, we identified 492 mRNAs with increased translation rates in dhh1 $1 \Delta$ cells, indicating that Dhh1 represses their translation (Supplemental Table S1). The identification of these mRNAs was not surprising as Dhh1 has been widely studied as a repressor of translation. Excitingly, we identified 538 additional mRNAs with decreased translation rates in dhh1 $\Delta$ cells, indicating that Dhh1 drives their translation in WT cells (Fig. 4B; Supplemental Table S1). Selected mRNAs from these two groups were validated to be translationally regulated by Dhh1 by Western blot and qPCR analysis (Supplemental Fig. S4A-C).

Dhh1 may drive translation of the identified mRNAs by a direct or indirect mechanism. To select the mRNAs directly interacting with Dhh1, we performed two independent CRAC experiments that showed a high correlation $(r=0.90)$ (Supplemental Fig. S5). In total, $183(37 \%)$ of the translationally repressed mRNAs and $245(46 \%)$ of the translationally activated mRNAs were specifically crosslinked to Dhh1 (Fig. 4C; Supplemental Table S1). Most mRNAs were crosslinked in the CDS and additionally in the 5' and/or 3' UTR (Fig. 4D; Supplemental Table S2). To test whether the Dhh1 crosslinking position defines its function in translation regulation, we determined the specific crosslinking sites for all crosslinked genes in the different subsets by calculating the CRAC read distribution across a virtual CDS (Fig. 4E) or per nucleotide (Supplemental Fig. S6). The mRNAs not translationally regulated by Dhh1 exhibit a low and largely uniformly distributed binding pattern. In contrast, mRNAs regulated by Dhh1 exhibit higher Dhh1 crosslinking, particularly around the initiation codon and before the stop codon, with crosslinking around the initiation codon much higher in translationally repressed mRNAs. These different crosslinking patterns may reflect the versatile function of Dhh1 in translational control.

Ribosome profiling enables detection of defects in specific translation steps. Ribosome pausing increases the likelihood of capturing a footprint by sequencing, and hence, defects in translation elongation will result in a peak in ribosome density. In contrast, defects in translation initiation will result in a lower RPF count throughout the complete CDS with no peaks in ribosome densities. We monitored ribosome pausing in different mRNA subsets by calculating the observed versus expected RPF reads (Ingolia et al. 2009) across a virtual CDS and plotted its distribution for the different sets of genes in WT and dhh1 $1 \Delta$ cells (Fig. 4F). Hereafter, "activated" only includes mRNAs translationally activated and crosslinked to Dhh1, "repressed," only mRNAs translationally repressed and crosslinked to Dhh1, and "unchanged," mRNAs with no changes at the mRNA and RPF level regardless of the crosslinking to Dhh1. In agreement 
A

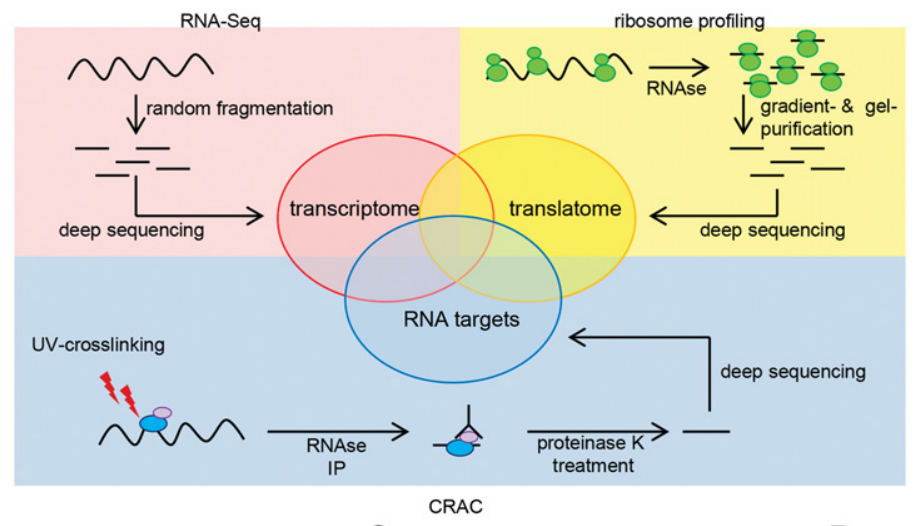

B

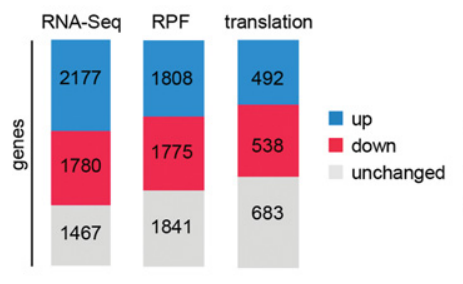

C

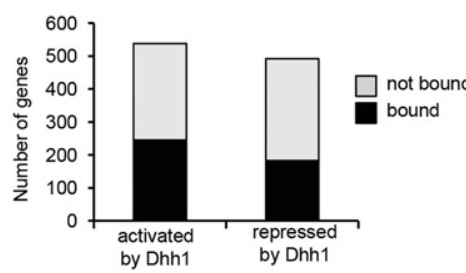

$\mathbf{E}$

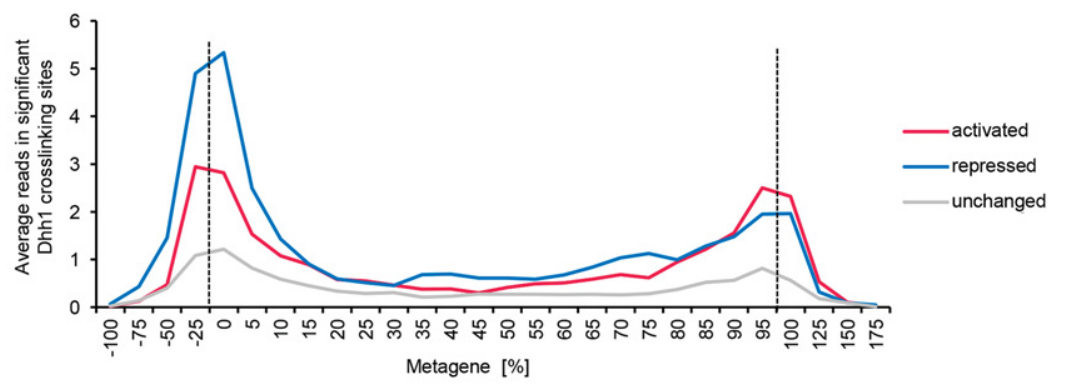

$\mathbf{F}$

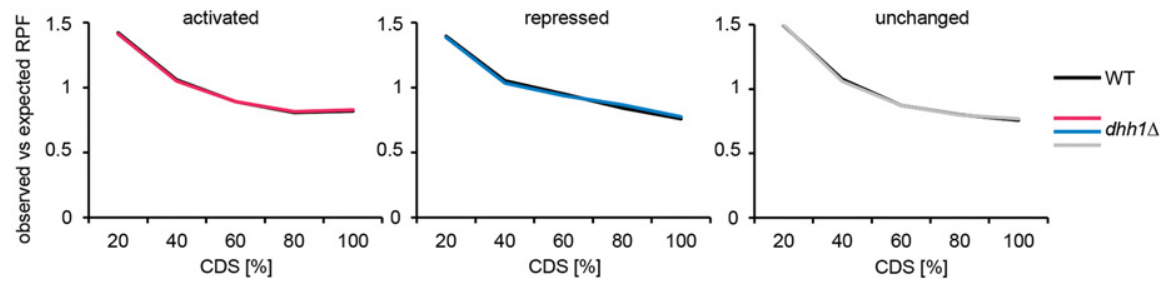

Figure 4. Dhh1 binds and regulates translation of distinct sets of cellular mRNAs. $(A)$ Experimental strategy to identify cellular mRNAs translationally controlled by Dhh1. (B) Changes in mRNA, RPF, and translation comparing WT to dhh1 $\Delta$ cells. Distribution of significant mRNA and RPF level changes is given for all genes and distribution of translation efficiency changes is given for genes with no changes at the mRNA level and for genes with opposite changes at the mRNA and RPF level. For RNA-seq data, "up" includes genes with a $\log _{2}$-fold change $>0.433$ and an adjusted $P$-value $<0.1$. "Down" includes genes with a $\log _{2}$-fold change $<-0.433$ and an adjusted $P$-value $<0.1$. For RPF data, "up" and "down" includes genes with a $\log _{2}$-fold change $>0$ and $<0$, respectively, and an adjusted $P$-value $<0.1$. (C) Bar graph showing the number of translationally activated and repressed genes crosslinked and not crosslinked by Dhh1. (D) Venn diagram depicting Dhh1 binding targets identified by CRAC in different transcript regions. ( $E$ ) Metagene analysis as indicated by CRAC data. $Y$-axis shows average number of reads in significant Dhh1 crosslinking sites (peaks) in the corresponding region. Reads that were not part of a significant peak are not considered. Dotted lines mark start and stop codon. $(F)$ Metagene analysis of ribosome density in WT and $d h h 1 \Delta$ cells for the different sets of mRNAs. $Y$-axis shows average of observed RPF reads related to expected ones.

with a role of Dhh1 on translation initiation, no significant differences were observed in WT and dhh1 $1 \Delta$ cells for any of the different subsets. Similar results were obtained when focusing on the first 120 nt versus the last ones (Supplemental Fig. S7).
D

Cellular mRNAs translationally activated by Dhhl contain long, highly structured CDSs

To further understand the underlying mechanism of Dhh1 function in translation regulation, we studied the physical properties of the specific mRNA subsets. First, we calculated the average length of the CDS and the $5^{\prime}$ and $3^{\prime}$ UTRs for mRNAs translationally activated, repressed, or not affected by Dhh1. Translationally activated mRNAs have, on average, a twofold longer CDS than repressed or not affected mRNAs (Fig. 5A). However, no significant length differences were detected between mRNA subsets for 5' and $3^{\prime}$ UTRs. It was previously shown that longer CDSs present lower ribosome density (Arava et al. 2003). This decreased translation efficiency might result from the fact that the folding probability increases with the length of the CDS. To explore this possibility, we calculated the intrinsic tendency to form secondary structures using the published parallel analyses of RNA structure (PARS) (Kertesz et al. 2010). In this pioneering study, PARS scores were obtained by coupling deep sequencing with enzyme probing of paired and unpaired nucleotides of isolated yeast total mRNAs. We calculated the mean PARS score for the CDS and the UTRs of the different mRNA subsets and "all" genes. "All" includes all mRNAs for which the PARS score has been published. Importantly, translationally activated mRNAs present higher mean PARS values in the CDS (0.49) than all genes (0.37) and higher than those mRNAs translationally repressed (0.33) or not affected (0.27), with these differences being highly significant $(P \leq 0.0001)$ (Fig. 5B). Noteworthy, both repressed and not affected genes have a lower mean PARS score in the $5^{\prime}$ UTR (0.05 and 0.08 , respectively) than all genes and translationally activated mRNAs (both 0.17). A plausible explanation for this observation would be that mRNAs not affected by Dhh1 do not require other RNA helicases, whereas the majority of all cellular mRNAs does require the action of RNA helicases. No significant differences were observed among PARS values of $3^{\prime}$ UTRs.

Next, we analyzed the PARS score distribution across a virtual gene in which values are averaged over percentage bins (25\% in the UTRs and 5\% in the CDS) for the three subsets of mRNAs and all mRNAs (Fig. 5C). Although mRNAs translationally activated by Dhh1 had significantly higher PARS values throughout the complete 5' UTR and CDS, one of the biggest

\section{Genome Research}

www.genome.org 
A
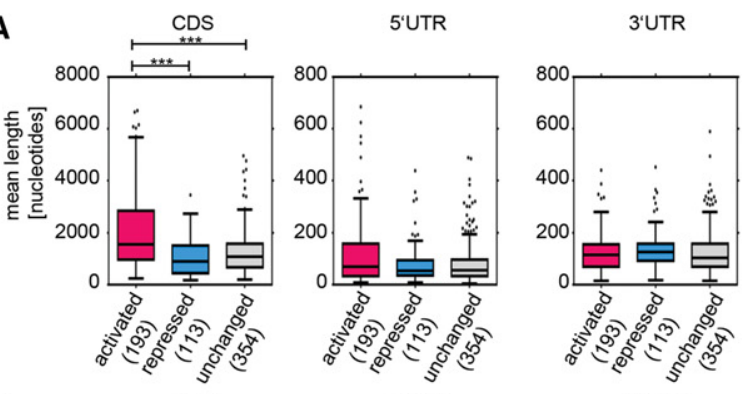

B
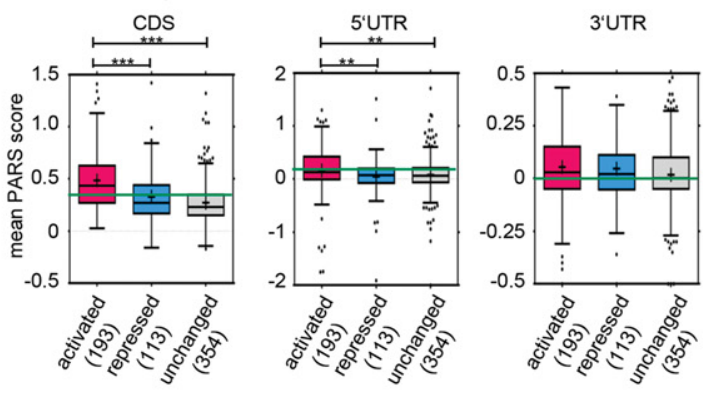

C

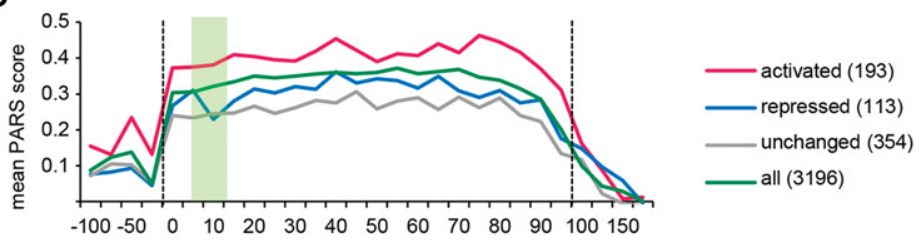

D

Metagene [\%]

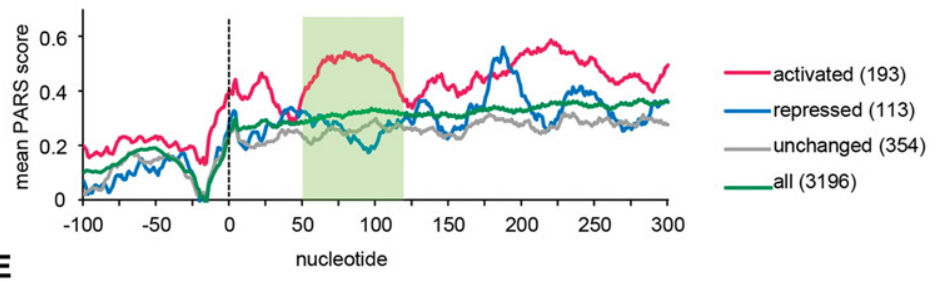

E

Biological process

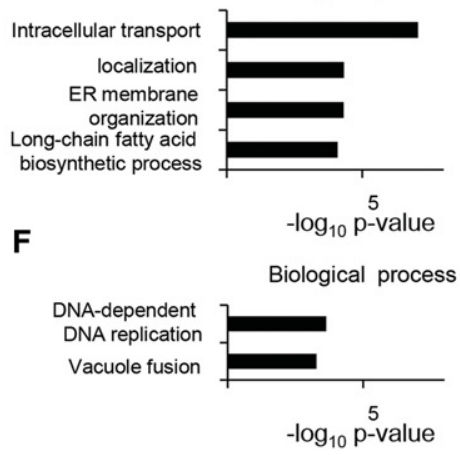

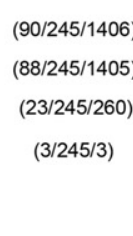

$(60 / 183 / 1133)$

(95/183/2127)

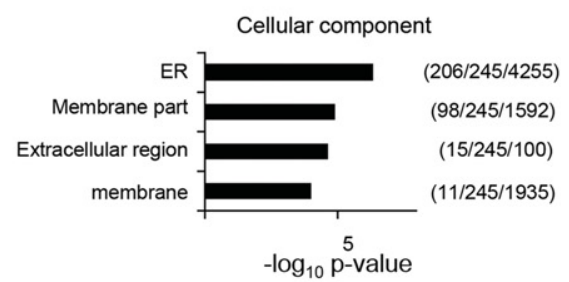

Cellular component

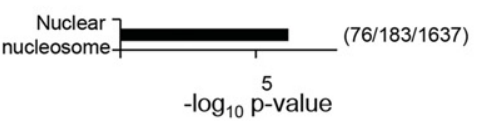

Figure 5. Cellular mRNAs translationally activated by Dhh1 contain highly structured CDSs. From the total number of mRNAs translationally activated, translationally repressed, and unchanged, only the given numbers were analyzed; as for the rest, the UTRs and CDSs are not annotated. ( $A$ ) Genes translationally activated by Dhh1 have a significantly longer $\operatorname{CDS}\left(P<0.0001\left[{ }^{* * *}\right]\right)$. $P$-values were computed after permuting randomly 1000 times the labels of the genes in the two groups (detailed in Supplemental Methods). Distribution of the average length of the $5^{\prime}$ UTR, CDS, and 3' UTR for genes translationally activated, repressed, and not affected by Dhh1 represented in a Tukey plot. Numbers in parentheses give the number of transcripts per subset. (B) Genes translationally activated by Dhh1 have significantly higher PARS scores in the CDS $\left(P<0.0001\left[{ }^{* * *}\right]\right)$ and the $5^{\prime}$ UTR $\left(P<0.01\left[{ }^{* *}\right]\right)$. $P$-values as in $A$. Distribution of the average PARS score of the $5^{\prime}$ UTR, CDS, and 3' UTR for genes translationally activated, repressed, and not affected by Dhh1 represented in a Tukey plot (band inside each box represents the median); cross indicates mean PARS score of each subset; green line marks mean PARS of all genes. Numbers in parentheses give the number of transcripts per subset. (C) Metagene PARS score analysis of the different sets of genes. $Y$-axis shows the average of smoothened PARS scores on the corresponding region. Smoothing is achieved by calculating the average PARS scores over a window of size 20 centered on the corresponding nucleotide. Dotted lines mark the start and stop codon; green box marks the $7.5 \%-15 \%$ region, one area with an especially high difference between the PARS scores. Numbers in the legend indicate the number of analyzed genes in each subset. (D) As in $C$, but for the $5^{\prime}$ UTR and the first $300 \mathrm{nt}$. Green box marks nucleotides 50-120. (E) GO term analysis for genes translationally activated and bound by Dhh1 regarding biological process and cellular component. Numbers in parentheses indicate $(B / n / b) . B=$ total number of genes associated with functional category (often comprising several GO terms grouped by REVIGO); $n=$ number of genes in input list; $b=$ number of genes in intersection. $(F)$ As in $E$, but for genes translationally repressed and bound by Dhh1. 
and more statistically significant differences between activated and repressed or not affected mRNAs was observed at the 7.5\%15\% region of the metagene (Fig. 5C; Supplemental Table S3A). To identify the nucleotides involved, we plotted the PARS score versus the nucleotide position (Fig. 5D). One of the biggest and more statistically significant differences between the activated and the repressed/unchanged mRNAs was found between nucleotides 50-120 after the initiation codon (Supplemental Table S3B). Remarkably, this area overlaps with the location of the mapped stem-loop in BMV RNA2 (nucleotides 42-87).

Together, these results show that Dhh1 is required to drive translation of a specific subset of cellular mRNAs that, similar to the BMV RNA, harbors highly structured 5' UTRs and long and highly structured CDSs.

\section{Dhhl drives translation of mRNAs coding primarily membrane and secreted proteins}

Specific mRNA features might allow coregulation of functionally related proteins. To determine whether the mRNAs translationally regulated by Dhh1 are functionally linked, we performed Gene Ontology (GO) analysis (Fig. 5E,F). Genes translationally activated by Dhh1 were highly enriched for intracellular transport processes. Accordingly, GO terms under cellular components were enriched in ER and membranes (Fig. 5E). In contrast, translationally repressed genes were enriched for proteins involved in DNA-dependent DNA replication and vacuole fusion. Accordingly, the GO term enriched regarding the cellular component of this subset was the nuclear nucleosome (Fig. 5F).

The ER and the cytosol represent distinct biological environments for translation with different regulatory factors affecting protein expression. Secreted proteins are preferentially translated at the ER by mechanisms that are still under intense study (Ast and Schuldiner 2013; Cui and Palazzo 2014; Jan et al. 2014; Reid and Nicchitta 2015). The canonical route is based on the cotranslational recognition of a hydrophobic region in the mRNA by the signal recognition particle (SRP). This results in a repression of translation until the RNP reaches the ER. Less-understood SRP-independent routes include ribosome-mediated mRNA targeting, post-translational targeting, and RNA-based localization (Ast and Schuldiner 2013). Based on published predictions (Ast et al. 2013), from the identified Dhh1-dependent mRNAs, 45\% utilize the SRP-dependent and 55\% a SRP-independent pathway, indicating that Dhh1-mediated translational control functions alongside the SRP-dependent and -independent pathways.

A previous study described that mRNAs encoding secreted proteins display lower PARS scores in the $5^{\prime}$ UTR and the first 30 nt of the CDS (Kertesz et al. 2010). This seems to be contradictory to our results, as the subset of activated genes consists of $\sim 30 \%$ of secreted proteins and is characterized by a higher PARS score. When we analyzed the PARS score distribution per metagene and per nucleotide of the secreted genes in the activated gene set (Supplemental Fig. S8A,B), we observed that these mRNAs exhibit an even higher PARS score in the $5^{\prime}$ UTR and CDS, including the first $30 \mathrm{nt}$, than those from the complete subset of translationally activated mRNAs. Thus, this specific group of secreted proteins harbors unique features that might correlate with their dependence on Dhh1 for translation.

\section{Dhhl functions as a translational activator in humans}

Dhh1 is highly conserved from yeast to humans. We have previously observed that DDX6, the Dhh1 human counterpart, drives translation of Hepatitis $C$ virus RNA in human hepatoma cell lines (Scheller et al. 2009). To approach whether this function is extended to human mRNAs, we first studied the physical properties of the human homologs of the yeast mRNAs translationally activated by Dhh1. They contained, like their yeast counterparts, long CDSs. In contrast to yeast, human mRNAs have been described not to contain, on average, highly structured CDSs (Wan et al. 2014). Interestingly, when we calculated the mean PARS score distribution using the PARS data set of the child described in Wan et al. (2014), we identified in the human mRNA homologs of the yeast mRNAs translationally activated by Dhh1, but not in the translationally repressed ones, structured regions in the CDSs. Interestingly, one of them is located 60-100 nt after the start codon (Fig. 6A), a location that overlaps with the one identified for yeast and viral mRNAs. Together, this suggests that DDX6 might drive translation of human mRNAs by similar mechanisms. As a proof-of-principle, we focused on the PTCH1 mRNA, the human counterpart of the yeast NCR1 mRNA, because of its clinical importance, as its de-regulation is a hallmark of pancreatic cancer. The PTCH1 mRNA exhibits a strong peak in the PARS score distribution after the starting codon (Fig. 6B) and has a very long CDS, features we defined to be typical for mRNAs translationally activated by Dhh1. Indeed, PTCH1 mRNA depended on DDX6 for translation. DDX6 depletion by siRNA-mediated silencing resulted in a decrease in the PTCH1 protein level without affecting the PTCH1 mRNA level, indicating a positive role of DDX6 in translation of the PTCH1 mRNA (Fig. 6C).

\section{Discussion}

The impact of RNA secondary and tertiary structures within the CDS on gene expression is poorly understood. Here, we describe a novel translation control mechanism, conserved from yeast to humans and hijacked by viruses, that involves the DEAD-box helicase Dhh1 and RNA structures within the UTRs and coding regions. Our results, first observed in a viral RNA and then extended to cellular mRNAs enriched in secreted proteins, are based on four major lines of evidence. First, the mRNAs present highly structured 5' UTRs and CDSs, including a structured region located around $70 \mathrm{nt}$ after the translation initiation site (nucleotides 42-87 for BMV RNA, nucleotides 50-120 for yeast mRNAs, and nucleotides 60-100 for human mRNAs). This was observed with PARS analyses and validated with mutational assays. Second, polysome and ribosome profiling suggest that Dhh1 drives translation at the translation initiation step. Consistent with a role in translation initiation, Dhh1 interacts with translation initiation factors, as observed in immunoprecipitation assays. Third, in contrast to its established role as a translational repressor and decapping activator, Dhh1 does not affect the steady-state level of the identified mRNAs, indicating that the role of Dhh1 in translation is not linked to its role in translation repression and decapping. Fourth, Dhh1 binds to mRNAs that depend on Dhh1 for translation with a different specificity compared to those mRNAs that do not depend on Dhh1 for translation, as found with CRAC analyses, suggesting a link between binding and mode of action. Together, these results uncover a novel translational regulation mechanism driven by Dhh1 that involves RNA folding within CDSs.

Using the ability of BMV RNA to translate and replicate in yeast, we find that not only the CDS but also both UTRs confer Dhh1-dependence for translation. Full dependence was only achieved when the three regions were present. This suggests a scenario in which Dhh1 remodels tertiary contacts involving

\section{Genome Research}

www.genome.org 


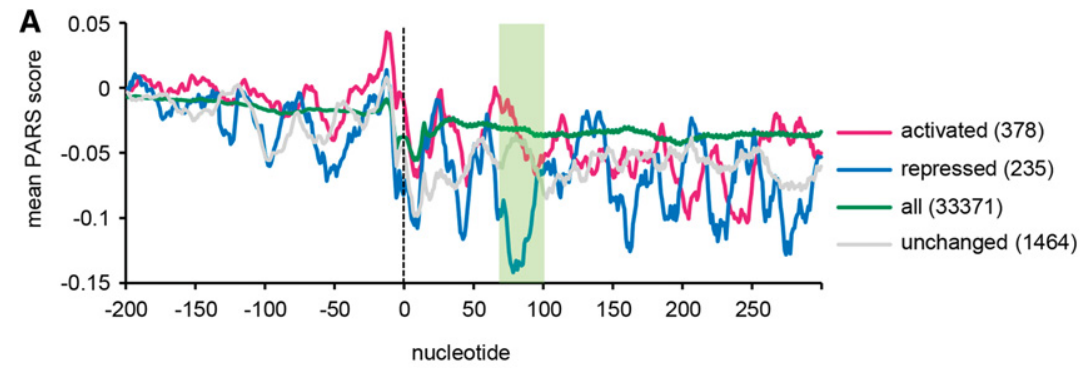

$\mathbf{B}$

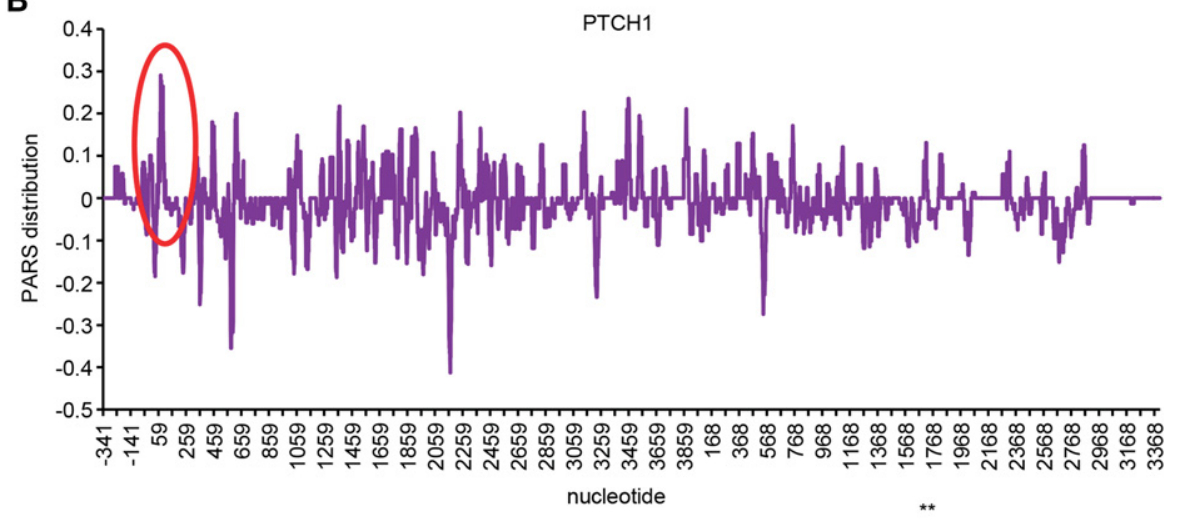

C
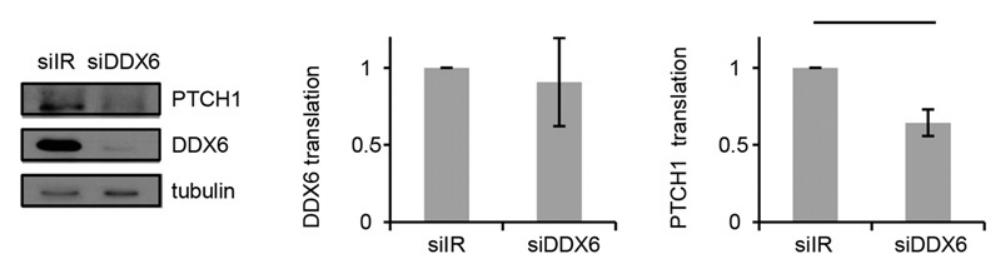

Figure 6. Dhh1-mediated translational control is seemingly conserved from yeast to humans. (A) PARS score distribution analysis of the human mRNA counterparts for the $5^{\prime}$ UTRs and the first 300 nt of the CDS. Axis and smoothing as in Figure 5C. Green box marks nucleotides 60-100. (B) PARS score of the $5^{\prime}$ UTR and the first $300 \mathrm{nt}$ of PTCH1 mRNA. (C) DDX6 drives translation of PTCH1. Western blot, qPCR, and translation rates of PTCH1 after DDX6 silencing. Translation was determined by correcting protein levels by the amount of the corresponding RNA. As a control for equal sample size, tubulin protein levels and HPRT1 mRNA levels were examined. Experiments have been carried out at least three times independently. $\left.{ }^{* *}\right) P<0.05$, t-test.

the three regions. Our CRAC data identified crosslinking of Dhh1 to sequences in the $3^{\prime}$ UTR and CDS but not to the identified stem-loop (Supplemental Fig. S1D). The low number of reads observed for the stem-loop sequence could be due to transient unwinding and therefore low steady-state interaction due to short residence of the helicase. Alternatively, Dhh1 might be required to remodel only some of the RNA helices involved in these interactions. In agreement with this model, a recent study shows that DEAD-box helicases can disrupt tertiary contacts by binding a secondary structure only after it spontaneously loses its tertiary contacts. After binding, they use ATP to unwind the helix (Pan et al. 2014). Interestingly, the requirement for spontaneous dynamics implies a preference of DEAD-box helicases for less stable RNA structures, which are likely to experience greater dynamic fluctuations (Pan et al. 2014). One would expect such low stable structures to exist in mRNA CDSs given that vastly fewer structures are identified by RNA probing in vivo than in vitro (Rouskin et al. 2014). Thus, a similar Dhh1 mode of action involving tertiary contacts might be operating the Dhh1-dependence of cellular mRNAs, given that they present high PARS values not only in the regions located around $70 \mathrm{nt}$ after the initiation codon but also across the CDSs and UTRs. Moreover, as for BMV RNA, we did not detect by CRAC analyses a direct Dhh1 binding to this region but to other sequences in the mRNA. Besides CDS RNA remodeling, Dhh1 might exert alternative or additional functions in translation. Our interesting observations that Dhh1 interacts with members of the eIF4F complex (Fig. 2E) and that both UTRs are involved in Dhh1-dependence suggest a model in which Dhh1 would help in forming a $5^{\prime}-3^{\prime}$ closed loop to favor translation. This might be of special importance for BMV RNA2 as it contains a tRNA-like structure at its $3^{\prime}$ end instead of a poly(A) tail, a major element that mediates circularization via PAB1 binding.

Given the high conservation of Dhh1 from yeast to mammals, it is plausible that its human counterpart DDX6 activates translation of certain human mRNAs by similar mechanisms. Indeed, we show that the human homologs of the yeast mRNAs translationally activated by Dhh1 share the highly structured region around nucleotide 70 of the CDS, and as a proof-of-principle, we demonstrated that DDX6 promotes translation of the PTCH1 mRNA. Given the role of DDX6 in PTCH1 mRNA translation, DDX6 overexpression would result in PTCH1 overexpression, a feature typical of pancreatic cancer. Interestingly, DDX6 is overexpressed in several cancers (Nakagawa et al. 1999; Hashimoto et al. 2001; Miyaji et al. 2003; Lin et al. 2008; Sen et al. 2015; Taniguchi et al. 2015), opening the possibility that translational regulation by DDX6 plays an important role in malignant transformations.

The cellular mRNAs translationally activated by Dhh1 encode mainly secreted and membrane proteins. Many of these mRNAs have in common that they are preferentially translated at the ER. Curiously, BMV RNA2 has been described to accumulate at the ER in the presence of the viral recruitment protein 1a, and Dhh1 assists in this recruitment process (Chen et al. 2001; Mas et al. 2006). Furthermore, deletion of a region in the RNA2 CDS containing the identified stem-loop decreases the recruitment rate (Chen et al. 2001). Similar to BMV, multiple positive-strand RNA viruses, including serious human pathogens such as HCV and the emerging Dengue virus and Chikungunya virus, replicate their RNA genome in ER-derived double membranes termed spherules (den Boon and Ahlquist 2010). The spherules function as organellelike compartments that scaffold, protect, and coordinate multiple facets of genome replication, expression, and encapsidation. Given that most viral infection steps are associated with ER membranes, it will seem plausible that these viruses would ensure expression of viral proteins in the ER to facilitate their function. 
Besides the presence of transmembrane domains in some viral proteins, little is known about how viral mRNAs would be preferentially translated at the ER. Our results suggest that positive-strand RNA viruses hijack Dhh1 not only to help in the recruitment process to the ER but also to promote translation of viral messengers through RNA remodeling.

Translation at the ER is under intense study as neither the targeting nor the translation mechanism is completely understood (Ast and Schuldiner 2013; for review, see Cui and Palazzo 2014; Reid and Nicchitta 2015). In our analyses, we identified 65 mRNAs coding secretome proteins (Ast et al. 2013) whose translation is promoted by Dhh1. Moreover, proximity-specific ribosome profiling analyses demonstrate that all these mRNAs are cotranslationally targeted to and translated at the ER (Jan et al. 2014). The targeting pathways involve SRP-dependent and SRP-independent mechanisms, suggesting that Dhh1 can function across pathways. Notably, the highly structured AUG-proximal CDS sequence located between nucleotides 50 and 120 in cellular mRNAs translationally activated by Dhh1 partially overlaps with two cis-acting ER targeting signals. One is the hydrophobic core domain recognized by SRP to halt translation (Meyer and Dobberstein 1980; Walter and Blobel 1981a,b; Walter et al. 1981; Meyer et al. 1982), and the other is a nonoptimal codon cluster of 35-40 codons located downstream from the SRP-binding site that promotes nascentchain recognition by slowing down translation (Pechmann et al. 2014). The location of these three features (SRP recognition site, nonoptimal codon cluster, and highly structured region) within the initial portion of the CDS highlights the complexity of the information stored in the mRNA sequence for translation. As secreted and membrane proteins often contain aggregation-prone hydrophobic domains, this complexity might be required to ensure appropriate local translation and to avoid accumulation of toxic aggregates in the cytosol. Interestingly, additional structural RNA cis-signals in the CDS might be guiding mRNA localization, as genome-wide structurome studies (Kertesz et al. 2010) identified an increased structure in the CDS of mRNAs encoding proteins that localize in specific subcellular compartments, such as cell wall, vacuole, and ER, or that function in distinct metabolic pathways, such as glycolysis, or organic acid and amine metabolic processes.

In our study, we also identified a group of mRNAs that are translationally repressed by Dhh1 and enriched in mRNAs coding proteins involved in nuclear processes. We were surprised by the relatively low number of translationally repressed mRNAs, as Dhh1 is well-known as a general translational repressor. However, of note is that in those studies Dhh1 was overexpressed or tethered to the $3^{\prime}$ UTR of an mRNA, or cells were stressed (Coller and Parker 2005; Carroll et al. 2011; Sweet et al. 2012), while in our study Dhh1 was depleted and cells were grown to log phase under normal growth conditions. Likewise, previous Dhh1 crosslinking and immunoprecipitation (CLIP) analyses, a UV crosslinking technique similar to CRAC, were carried out under stress conditions (Mitchell et al. 2013). Despite these differences, 58\% of the identified Dhh1-bound mRNAs are common in both conditions.

In conclusion, our findings bring to light a novel layer of translational control that involves the DEAD-box RNA helicase Dhh1 and RNA folding within the CDS. Two other DEAD-box helicases, Ded1 and eIF4A, have recently been shown to jointly facilitate ribosome scanning through secondary structures at the $5^{\prime}$ UTR of mRNAs (Sen et al. 2015). Thus, a complex cooperation between distinct DEAD-box helicases seems to be required for trans- lation to proceed, possibly allowing tight control over specific mRNA fates.

\section{Methods}

Yeast strains, molecular and cell biology techniques, a list of plasmids, high-throughput methods, and detailed computational analysis are described in Supplemental Methods. Unless indicated, all yeast growth and translation experiments were carried out under permissive conditions $\left(30^{\circ} \mathrm{C}\right)$.

\section{Polysome profiling}

Polysome profiling was carried out as previously described (Noueiry et al. 2003) with slight modifications. Briefly, cells were grown from $\mathrm{OD}_{600}=0.02$ to mid-exponential phase $\left(\mathrm{OD}_{600} \sim\right.$ 0.4 ) at $30^{\circ} \mathrm{C}$ and treated for $1 \mathrm{~min}$ with $100 \mu \mathrm{g} / \mathrm{mL}$ cyclohexamide (CHX). Cells were harvested by vacuum filtration, frozen in liquid nitrogen, and pulverized under cryogenic conditions at $5 \mathrm{cps}$ in a SPEX 6750 Freezer/Mill (SPEX SamplePrep) in $20 \mathrm{mM}$ Tris- $\mathrm{HCl}$ ( $\mathrm{pH}=7.5), 100 \mathrm{mM} \mathrm{NaCl}, 10 \mathrm{mM} \mathrm{MgCl} 2,1 \%$ Triton X-100, 0.5 $\mathrm{mM}$ DTT, $100 \mu \mathrm{g} / \mathrm{mL} \mathrm{CHX}$. Extracts were purified by several centrifugation steps. Twelve units of $\mathrm{OD}_{260}$ from extracts were loaded on a linear 10\%-50\% sucrose gradient and spun for $3 \mathrm{~h}$ at 35,000 rpm in a Beckman SW41 rotor. Fractions were collected, and $30 \mathrm{ng}$ of purified BMV RNA3 were added to each fraction before further processing to normalize for technical variations during the purification. RNA was purified and analyzed by Northern blot.

\section{Ribosome profiling}

Ribosome profiling was carried out as previously described (Ingolia et al. 2009, 2012; Nedialkova and Leidel 2015). Detailed information of the experimental procedure and details on ribosome profiling data analysis is available in the Supplemental Methods.

\section{CRAC experiments and analysis}

CRAC experiments were performed as previously described (Bohnsack et al. 2009, 2012) and analyzed using pyicoclip (http://regulatorygenomics.upf.edu/Software/Pyicoteo/pyicoclip. $\mathrm{html})$. Detailed processing information is available in the Supplemental Methods.

\section{RNA secondary structure prediction}

All RNA secondary structure prediction and base-pairing probability calculations were carried out by running RNAfold and RNAfold -p from the Vienna package, version 2.1.7 (Lorenz et al. 2011). Design of the mutant RNA2-BMV stem-loop was carried out using RNAiFold (Garcia-Martin et al. 2013).

\section{Data access}

The ribosome profiling and RNA-seq data from $d h h 1 \Delta$ cells in this study have been submitted to the NCBI Gene Expression Omnibus (GEO; http://www.ncbi.nlm.nih.gov/geo/) under accession number GSE87892. The CRAC data from this study have been submitted to GEO under accession number GSE87888.

\section{Acknowledgments}

We thank P. Carvalho, R. Bock, R. Lill, Arlen Johnson, and P. Ahlquist for reagents. We thank K. Qu and H. Chang for the processed human PARS data. We also thank B. Blasco, F. Gebauer, R. Méndez, and A. Palazzo for stimulating discussions and

\section{Genome Research}

www.genome.org 
comments on the manuscript and P. Hackert for help with the CRAC experiments. This work was supported by the Spanish Ministry of Economy and Competitiveness through grants BFU2013-44629-R, BFU2016-80039-R (AEI/MINEICO/FEDER, UE) and the "Maria de Maeztu" Programme for Units of Excellence in R\&D (MDM-2014-0370). J.J. was supported by the grant 2012FI_B00574 from the Generalitat de Catalunya. This work was also supported by the Deutsche Forschungsgemeinschaft (SFB860 to M.T.B.), the Alexander von Humboldt foundation (to K.E.S. and M.T.B.), the Max Planck Society (to S.A.L.), the Spanish Ministerio de Economia y Competividad/ISCIII-FEDER (PI14/00125 to P.N.), and the Generalitat de Catalunya (2014/SGR/143 to P.N.).

\section{References}

Alves-Rodrigues I, Mas A, Diez J. 2007. Xenopus Xp54 and human RCK/p54 helicases functionally replace yeast Dhh1p in Brome mosaic virus RNA replication. J Virol 81: 4378-4380.

Arava Y, Wang Y, Storey JD, Liu CL, Brown PO, Herschlag D. 2003. Genomewide analysis of mRNA translation profiles in Saccharomyces cerevisiae. Proc Natl Acad Sci 100: 3889-3894.

Ast T, Schuldiner M. 2013. All roads lead to Rome (but some may be harder to travel): SRP-independent translocation into the endoplasmic reticulum. Crit Rev Biochem Mol Biol 48: 273-288.

Ast T, Cohen G, Schuldiner M. 2013. A network of cytosolic factors targets SRP-independent proteins to the endoplasmic reticulum. Cell 152: 1134-1145.

Bohnsack MT, Martin R, Granneman S, Ruprecht M, Schleiff E, Tollervey D. 2009. Prp43 bound at different sites on the pre-rRNA performs distinct functions in ribosome synthesis. Mol Cell 36: 583-592.

Bohnsack MT, Tollervey D, Granneman S. 2012. Identification of RNA helicase target sites by UV cross-linking and analysis of cDNA. Methods Enzymol 511: 275-288.

Carroll JS, Munchel SE, Weis K. 2011. The DExD/H box ATPase Dhh1 functions in translational repression, mRNA decay, and processing body dynamics. J Cell Biol 194: 527-537.

Chen J, Noueiry A, Ahlquist P. 2001. Brome mosaic virus protein 1a recruits viral RNA2 to RNA replication through a 5' proximal RNA2 signal. J Virol 75: 3207-3219.

Chen Y, Boland A, Kuzuoglu-Ozturk D, Bawankar P, Loh B, Chang CT, Weichenrieder O, Izaurralde E. 2014. A DDX6-CNOT1 complex and W-binding pockets in CNOT9 reveal direct links between miRNA target recognition and silencing. Mol Cell 54: 737-750.

Cheng Z, Coller J, Parker R, Song H. 2005. Crystal structure and functional analysis of DEAD-box protein Dhh1p. RNA 11: 1258-1270.

Chu CY, Rana TM. 2006. Translation repression in human cells by microRNA-induced gene silencing requires RCK/p54. PLoS Biol 4: e210.

Coller J, Parker R. 2005. General translational repression by activators of mRNA decapping. Cell 122: 875-886.

Coller JM, Tucker M, Sheth U, Valencia-Sanchez MA, Parker R. 2001. The DEAD box helicase, Dhh1p, functions in mRNA decapping and interacts with both the decapping and deadenylase complexes. RNA 7: 1717-1727.

Cui XA, Palazzo AF. 2014. Localization of mRNAs to the endoplasmic reticulum. Wiley Interdiscip Rev RNA 5: 481-492.

Cullen BR. 2009. Viral RNAs: lessons from the enemy. Cell 136: 592-597.

den Boon JA, Ahlquist P. 2010. Organelle-like membrane compartmentalization of positive-strand RNA virus replication factories. Annu Rev Microbiol 64: 241-256.

Dutta A, Zheng S, Jain D, Cameron CE, Reese JC. 2011. Intermolecular interactions within the abundant DEAD-box protein Dhh1 regulate its activity in vivo. J Biol Chem 286: 27454-27470.

Franks TM, Lykke-Andersen J. 2008. The control of mRNA decapping and Pbody formation. Mol Cell 32: 605-615.

Garcia-Martin JA, Clote P, Dotu I. 2013. RNAiFold: a web server for RNA inverse folding and molecular design. Nucleic Acids Res 41: W465-W470.

Haimovich G, Medina DA, Causse SZ, Garber M, Millan-Zambrano G, Barkai O, Chavez S, Perez-Ortin JE, Darzacq X, Choder M. 2013. Gene expression is circular: Factors for mRNA degradation also foster mRNA synthesis. Cell 153: 1000-1011.

Hashimoto K, Nakagawa Y, Morikawa H, Niki M, Egashira Y, Hirata I, Katsu K, Akao Y. 2001. Co-overexpression of DEAD box protein rck/p54 and cmyc protein in human colorectal adenomas and the relevance of their expression in cultured cell lines. Carcinogenesis 22: 1965-1970.

Huys A, Thibault PA, Wilson JA. 2013. Modulation of hepatitis C virus RNA accumulation and translation by DDX6 and miR-122 are mediated by separate mechanisms. PLoS One 8: e67437.
Ingolia NT, Ghaemmaghami S, Newman JR, Weissman JS. 2009. Genomewide analysis in vivo of translation with nucleotide resolution using ribosome profiling. Science 324: 218-223.

Ingolia NT, Brar GA, Rouskin S, McGeachy AM, Weissman JS. 2012. The ribosome profiling strategy for monitoring translation in vivo by deep sequencing of ribosome-protected mRNA fragments. Nat Protoc 7: $1534-1550$.

Jan CH, Williams CC, Weissman JS. 2014. Principles of ER cotranslational translocation revealed by proximity-specific ribosome profiling. Science 346: 1257521.

Jarmoskaite I, Russell R. 2014. RNA helicase proteins as chaperones and remodelers. Annu Rev Biochem 83: 697-725.

Kertesz M, Wan Y, Mazor E, Rinn JL, Nutter RC, Chang HY, Segal E. 2010. Genome-wide measurement of RNA secondary structure in yeast. Nature 467: 103-107.

Lin F, Wang R, Shen JJ, Wang X, Gao P, Dong K, Zhang HZ. 2008. Knockdown of RCK/p54 expression by RNAi inhibits proliferation of human colorectal cancer cells in vitro and in vivo. Cancer Biol Ther 7: 1669-1676.

Linder P, Jankowsky E. 2011. From unwinding to clamping - the DEAD box RNA helicase family. Nat Rev Mol Cell Biol 12: 505-516.

Lorenz R, Bernhart SH, Honer Zu Siederdissen C, Tafer H, Flamm C, Stadler PF, Hofacker IL. 2011. ViennaRNA Package 2.0. Algorithms Mol Biol 6: 26.

Maekawa H, Nakagawa T, Uno Y, Kitamura K, Shimoda C. 1994. The ste13+ gene encoding a putative RNA helicase is essential for nitrogen starvation-induced G1 arrest and initiation of sexual development in the fission yeast Schizosaccharomyces pombe. Mol Gen Genet 244: 456-464.

Martin R, Straub AU, Doebele C, Bohnsack MT. 2013. DExD/H-box RNA helicases in ribosome biogenesis. RNA Biol 10: 4-18.

Mas A, Alves-Rodrigues I, Noueiry A, Ahlquist P, Diez J. 2006. Host deadenylation-dependent mRNA decapping factors are required for a key step in brome mosaic virus RNA replication. J Virol 80: 246-251.

Mathys H, Basquin J, Ozgur S, Czarnocki-Cieciura M, Bonneau F, Aartse A, Dziembowski A, Nowotny M, Conti E, Filipowicz W. 2014. Structural and biochemical insights to the role of the CCR4-NOT complex and DDX6 ATPase in microRNA repression. Mol Cell 54: 751-765.

Melcher U. 1971. Metabolism of puromycin by yeast cells. Biochim Biophys Acta 246: $216-224$.

Meyer DI, Dobberstein B. 1980. Identification and characterization of a membrane component essential for the translocation of nascent proteins across the membrane of the endoplasmic reticulum. J Cell Biol 87: 503-508.

Meyer DI, Krause E, Dobberstein B. 1982. Secretory protein translocation across membranes-the role of the 'docking protein'. Nature 297: 647-650.

Mitchell SF, Jain S, She M, Parker R. 2013. Global analysis of yeast mRNPs. Nat Struct Mol Biol 20: 127-133.

Miyaji K, Nakagawa Y, Matsumoto K, Yoshida H, Morikawa H, Hongou Y, Arisaka Y, Kojima H, Inoue T, Hirata I, et al. 2003. Overexpression of a DEAD box/RNA helicase protein, rck/p54, in human hepatocytes from patients with hepatitis $C$ virus-related chronic hepatitis and its implication in hepatocellular carcinogenesis. J Viral Hepat 10: 241-248.

Mortimer SA, Kidwell MA, Doudna JA. 2014. Insights into RNA structure and function from genome-wide studies. Nat Rev Genet 15: 469-479.

Nakagawa Y, Morikawa H, Hirata I, Shiozaki M, Matsumoto A, Maemura K, Nishikawa T, Niki M, Tanigawa N, Ikegami M, et al. 1999. Overexpression of rck/p54, a DEAD box protein, in human colorectal tumours. Br J Cancer 80: 914-917.

Nedialkova DD, Leidel SA. 2015. Optimization of codon translation rates via tRNA modifications maintains proteome integrity. Cell 161: 1606-1618.

Nissan T, Rajyaguru P, She M, Song H, Parker R. 2010. Decapping activators in Saccharomyces cerevisiae act by multiple mechanisms. Mol Cell 39: $773-783$.

Noueiry AO, Ahlquist P. 2003. Brome mosaic virus RNA replication: revealing the role of the host in RNA virus replication. Annu Rev Phytopathol 41: 77-98.

Noueiry AO, Diez J, Falk SP, Chen J, Ahlquist P. 2003. Yeast Lsm1p-7p/Pat1p deadenylation-dependent mRNA-decapping factors are required for brome mosaic virus genomic RNA translation. Mol Cell Biol 23: 4094-4106.

Pan C, Potratz JP, Cannon B, Simpson ZB, Ziehr JL, Tijerina P, Russell R. 2014. DEAD-box helicase proteins disrupt RNA tertiary structure through helix capture. PLoS Biol 12: e1001981.

Pechmann S, Chartron JW, Frydman J. 2014. Local slowdown of translation by nonoptimal codons promotes nascent-chain recognition by SRP in vivo. Nat Struct Mol Biol 21: 1100-1105.

Presnyak V, Coller J. 2013. The DHH1/RCKp54 family of helicases: an ancient family of proteins that promote translational silencing. Biochim Biophys Acta 1829: 817-823. 
Reid DW, Nicchitta CV. 2015. Diversity and selectivity in mRNA translation on the endoplasmic reticulum. Nat Rev Mol Cell Biol 16: 221-231.

Rouskin S, Zubradt M, Washietl S, Kellis M, Weissman JS. 2014. Genomewide probing of RNA structure reveals active unfolding of mRNA structures in vivo. Nature 505: 701-705.

Scheller N, Mina LB, Galao RP, Chari A, Gimenez-Barcons M, Noueiry A, Fischer U, Meyerhans A, Diez J. 2009. Translation and replication of hepatitis $C$ virus genomic RNA depends on ancient cellular proteins that control mRNA fates. Proc Natl Acad Sci 106: 13517-13522.

Schindler D, Davies J. 1975. Inhibitors of macromolecular synthesis in yeast. Methods Cell Biol 12: 17-38.

Sen ND, Zhou F, Ingolia NT, Hinnebusch AG. 2015. Genome-wide analysis of translational efficiency reveals distinct but overlapping functions of yeast DEAD-box RNA helicases Ded1 and eIF4A. Genome Res 25: 1196-1205.

Sweet T, Kovalak C, Coller J. 2012. The DEAD-box protein Dhh1 promotes decapping by slowing ribosome movement. PLoS Biol 10: e1001342.

Takyar S, Hickerson RP, Noller HF. 2005. mRNA helicase activity of the ribosome. Cell 120: 49-58.

Taniguchi K, Sugito N, Kumazaki M, Shinohara H, Yamada N, Matsuhashi N, Futamura M, Ito Y, Otsuki Y, Yoshida K, et al. 2015. Positive feedback of DDX6/c-Myc/PTB1 regulated by miR-124 contributes to maintenance of the Warburg effect in colon cancer cells. Biochim Biophys Acta 1852: 1971-1980.

Thermann R, Hentze MW. 2007. Drosophila miR2 induces pseudo-polysomes and inhibits translation initiation. Nature 447: 875-878.
Tseng-Rogenski SS, Chong JL, Thomas CB, Enomoto S, Berman J, Chang TH. 2003. Functional conservation of Dhh1p, a cytoplasmic DExD/H box protein present in large complexes. Nucleic Acids Res 31: 4995-5002.

Walter P, Blobel G. 1981a. Translocation of proteins across the endoplasmic reticulum III. Signal recognition protein (SRP) causes signal sequencedependent and site-specific arrest of chain elongation that is released by microsomal membranes. J Cell Biol 91: 557-561.

Walter P, Blobel G. 1981b. Translocation of proteins across the endoplasmic reticulum. II. Signal recognition protein (SRP) mediates the selective binding to microsomal membranes of in-vitro-assembled polysomes synthesizing secretory protein. J Cell Biol 91: 551-556.

Walter P, Ibrahimi I, Blobel G. 1981. Translocation of proteins across the endoplasmic reticulum. I. Signal recognition protein (SRP) binds to in-vitro-assembled polysomes synthesizing secretory protein. J Cell Biol 91: $545-550$.

Wan Y, Qu K, Zhang QC, Flynn RA, Manor O, Ouyang Z, Zhang J, Spitale RC, Snyder MP, Segal E, et al. 2014. Landscape and variation of RNA secondary structure across the human transcriptome. Nature 505: 706-709.

Westmoreland TJ, Olson JA, Saito WY, Huper G, Marks JR, Bennett CB 2003. DHH1 regulates the G1/S-checkpoint following DNA damage or BRCA1 expression in yeast. J Surg Res 113: 62-73.

Received April 26, 2016; accepted in revised form November 3, 2016.

\section{Genome Research}




\section{Corrigendum}

Genome Research 27: 95-106 (2017)

Corrigendum: A novel translational control mechanism involving RNA structures within coding sequences

Jennifer Jungfleisch, Danny D. Nedialkova, Ivan Dotu, Katherine E. Sloan, Neus Martinez-Bosch, Lukas Brüning, Emanuele Raineri, Pilar Navarro, Markus T. Bohnsack, Sebastian A. Leidel, and Juana Díez

The authors would like to correct the omission of a funding number in the Acknowledgments section. The fourth sentence of that paragraph should read as follows: "This work was supported by the Spanish Ministry of Economy and Competitiveness through grants BFU2013-44629-R, BFU2016-80039-R (AEI/ MINEICO/FEDER, UE) and the "Maria de Maeztu" Programme for Units of Excellence in R\&D (MDM-20140370)." The authors apologize for this omission. The sentence has already been corrected in both the PDF and full-text files online.

doi: $10.1101 /$ gr.222133.117 


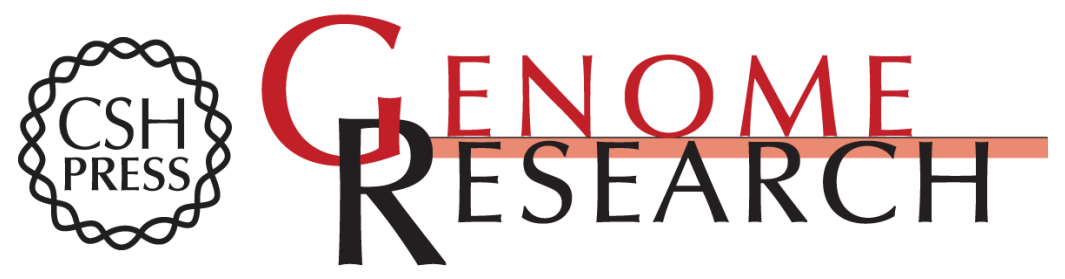

\section{A novel translational control mechanism involving RNA structures within coding sequences}

Jennifer Jungfleisch, Danny D. Nedialkova, Ivan Dotu, et al.

Genome Res. 2017 27: 95-106 originally published online November 7, 2016

Access the most recent version at doi:10.1101/gr.209015.116

\section{Supplemental Material \\ Related Content}

References

This article cites 63 articles, 17 of which can be accessed free at:

http://genome.cshlp.org/content/27/1/95.full.html\#ref-list-1

Articles cited in:

http://genome.cshlp.org/content/27/1/95.full.html\#related-urls

Corrigendum: A novel translational control mechanism involving RNA Jennifer Jungfleisch, Danny D. Nedialkova, Ivan Dotu, et al. Genome Res. April , 2017 27: 663

Creative Commons

This article is distributed exclusively by Cold Spring Harbor Laboratory Press for the first six months after the full-issue publication date (see

License

http://genome.cshlp.org/site/misc/terms.xhtml). After six months, it is available under a Creative Commons License (Attribution-NonCommercial 4.0 International), as described at http://creativecommons.org/licenses/by-nc/4.0/.

Email Alerting Receive free email alerts when new articles cite this article - sign up in the box at the Service top right corner of the article or click here.

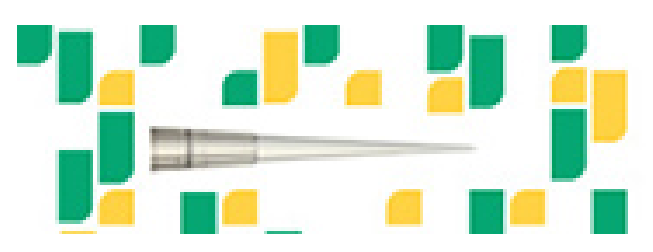

Focused on your science.

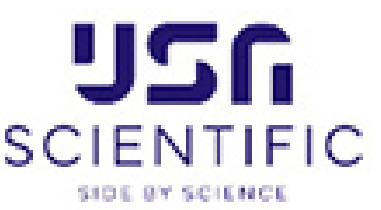

To subscribe to Genome Research go to:

https://genome.cshlp.org/subscriptions 